\title{
Detection of Right Coronary Artery Air Embolism by Transesophageal Echocardiography
}

\author{
${ }^{1}$ Krishna P Gourav, ${ }^{2}$ Imran Bhat, ${ }^{3}$ Srinath Damodaran , ${ }^{4}$ Sunderlal Negi, ${ }^{5}$ Gurpinder S Ghotra
}

\begin{abstract}
Cardiac air embolism is common while undergoing cardiac surgeries as these require an opening of left-sided cardiac chambers, right-sided cardiac chambers, aorta or due to inadequate de-airing of a saphenous vein graft. Right coronary artery air embolism is one of the common cause of hemodynamic instability after coming off cardiopulmonary bypass. We discussed two cases in which the transesophageal echocardiography (TEE) helped in diagnosing right-coronary artery air embolism as the cause of sudden ST-segment elevation, hemodynamic instability, and right ventricular (RV) dysfunction.
\end{abstract}

Keywords: Intracoronary air embolism, Right ventricle dysfunction, Transesophageal echocardiography.

How to cite this article: Gourav KP, Bhat I, Damodaran S, Negi S, Ghotra GS. Detection of Right Coronary Artery Air Embolism by Transesophageal Echocardiography. J Perioper Echocardiogr 2018;6(1):29-32.

Source of support: Nil

Conflict of interest: None

\section{CASE REPORT}

\section{Patient 1}

A 60-year-old man weighing $73 \mathrm{~kg}$ presented to our institute with complaints of chest pain on exertion and grade 2 dyspnea from 4 months. On examination, his blood pressure was $150 / 70 \mathrm{~mm} \mathrm{Hg}$ and heart rate was $67 / \mathrm{min}$. The TTE showed a regional wall motion abnormality (RWMA) in left anterior descending (LAD) artery tertiary (mid-anterior and mid anteroseptal segments) and left circumflex (LCX) artery territory (mid anterolateral segment) with mild left ventricular (LV) dysfunction. It also showed mild aortic and mitral regurgitation. Further, coronary angiography revealed 90\% stenosis in LAD artery, 90\% stenosis in LCX artery, $40 \%$ stenosis in right coronary artery (RCA), 30\% stenosis in a posterior descending artery, $40 \%$ stenosis in obtuse

\footnotetext{
1-3,5Senior Resident, ${ }^{4}$ Assistant Professor

${ }^{1-5}$ Department of Anesthesia and Intensive Care, Postgraduate Institute of Medical Education and Research, Chandigarh, India

Corresponding Author: Krishna P Gourav, Senior Resident, Department of Anesthesia and Intensive Care, Postgraduate Institute of Medical Education and Research, Chandigarh, India, e-mail: kpgmbbs777@gmail.com
}

marginal and 30\% stenosis in a 1st diagonal branch of LAD. Hence, coronary artery bypass grafting (CABG) surgery was planned. TEE, using a GE-6VT adult probe, was performed in the operating room after induction of anesthesia and TTE findings were confirmed. After adequate heparinization, cardiopulmonary bypass (CPB) was instituted after inserting two stages cavoatrial venous cannula and ascending aorta arterial cannula. Under CPB, three grafts were performed, i.e. left internal mammary artery to LAD artery, great saphenous vein grafts to the LCX artery and diagonal branch (D1) of LAD artery. After CABG, the patient was successfully weaned off from CPB using milrinone $0.3 \mathrm{mcg} / \mathrm{kg} /$ $\mathrm{min}$ and nor-adrenaline $0.05 \mathrm{mcg} / \mathrm{kg} / \mathrm{min}$. The TEE was used to inspect the adequacy of de-airing before coming off $\mathrm{CPB}$. However, in spite of that, a pocket of air was still present on the inter-atrial septum towards the left atrium and in the right upper pulmonary vein (Fig. 1 and Video 1). In addition to that, it revealed a normal biventricular function with the disappearance of RWMA in LAD and LCX territory. Five minutes after termination of $\mathrm{CPB}$, the patient suddenly became hemodynamically unstable with ST-segment elevation in inferior electrocardiographic (ECG) leads (II, III, aVF) followed by ventricular fibrillation (VF). On careful examination of aortic sinus in TEE mid esophageal aortic valve long axis (ME LAX) view, we observed air bubbles entering into the right coronary artery (Fig. 2 and Video 2).

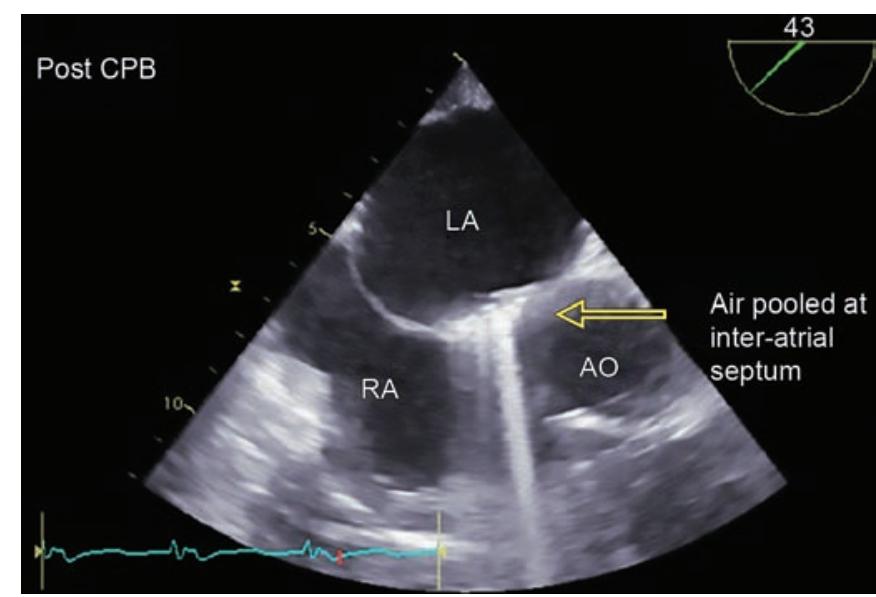

Fig. 1: Mid esophageal right ventricle inflow-outflow view showing air pooled on the inter-atrial septum towards left atrium after cardiopulmonary bypass ( $L A$, left atrium; RA, right atrium; $A O$, aorta) 


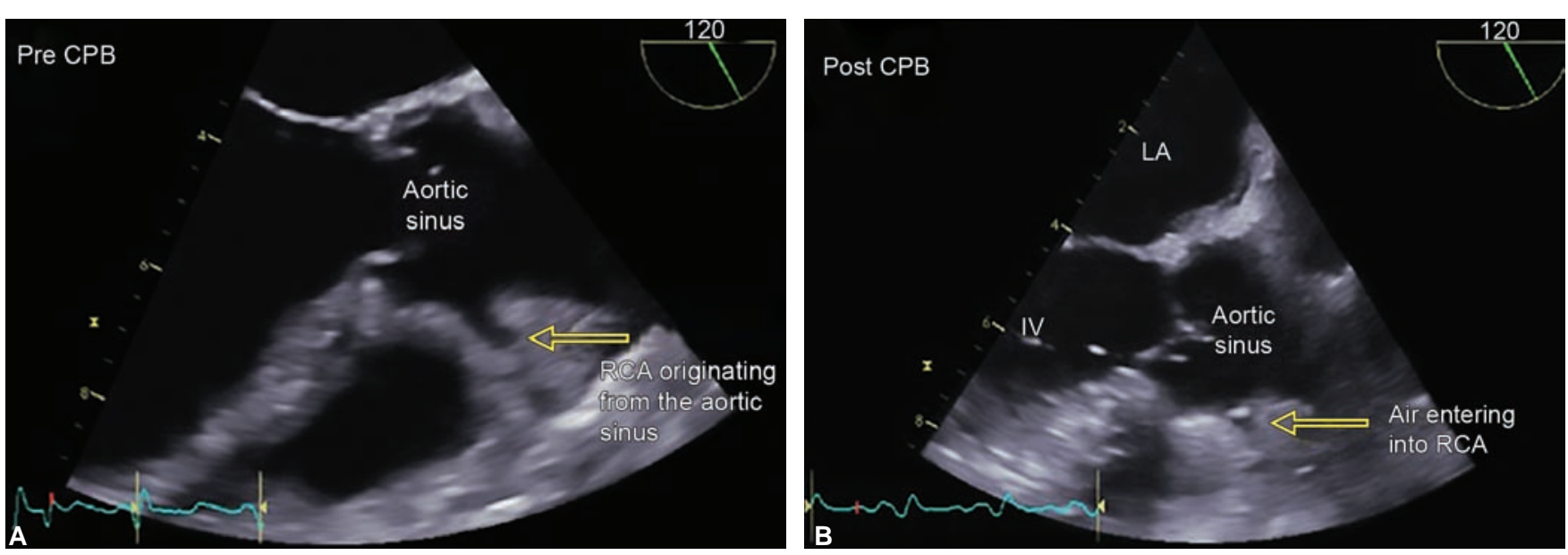

Figs 2A and B: Mid esophageal aortic valve long axis view showing $(A)$ the right coronary artery (RCA) before cardiopulmonary bypass (CPB); (B) air entering into the RCA after CPB. (LA, left atrium; LV, left ventricle; RV)
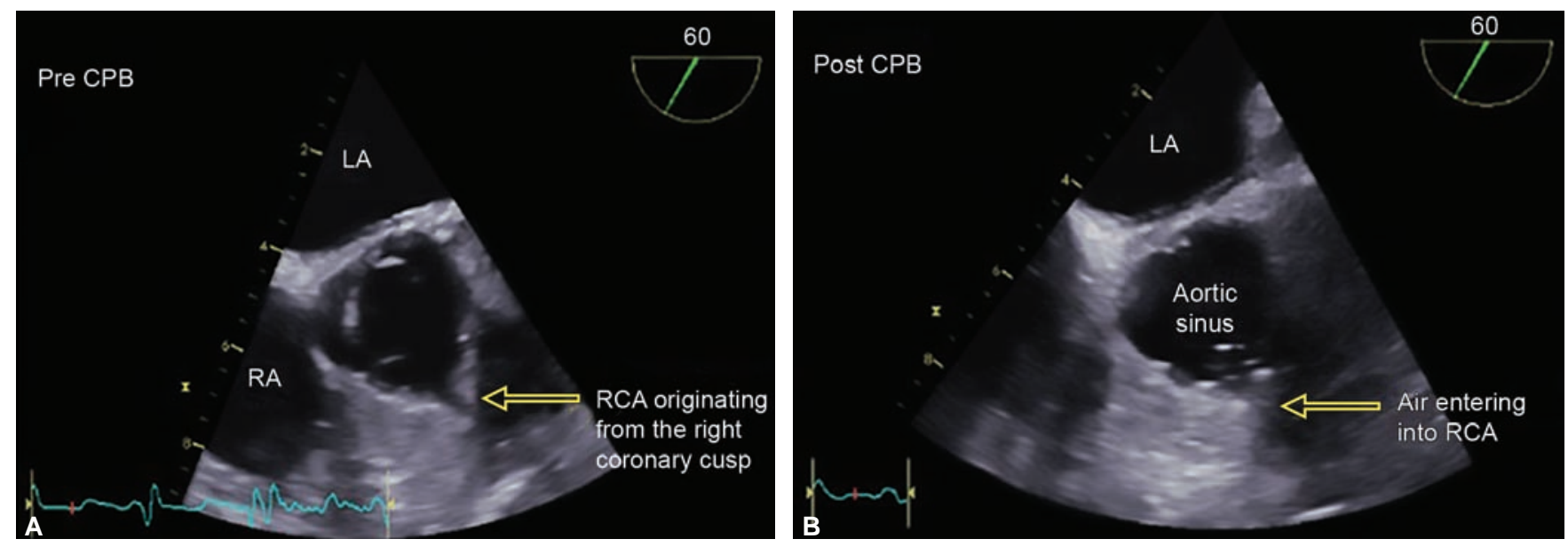

Figs 3A and B: Mid esophageal aortic valve short axis view showing $(A)$ the right coronary artery (RCA) before cardiopulmonary bypass (CPB); (B) air entering into the RCA after CPB. (LA, left atrium; RA, right atrium)

Similarly, on aortic valve short axis view we observed the same findings (Fig. 3), thus helping in diagnosing the cause of hemodynamic instability. Additionally, right ventricular (RV) systolic dysfunction (RV fractional area change $22 \%$; tricuspid annular plane systolic excursion of $10 \mathrm{~mm}$ ) and hypokinesia in inferior wall of LV (mid inferior and apical inferior segments) noted in TEE were supportive of RCA air embolism. The CPB was reinstituted, aortic root vent was reinserted and started at $250 \mathrm{~mL} /$ minute, coronary perfusion pressure was increased by increasing systemic mean arterial pressure after giving boluses of vasopressors. After thorough de-airing and improved systemic perfusion pressure, RV function and ECG returned to baseline, and the air pocket in the inter-atrial septum disappeared. The biventricular function recovered without any further sequelae. The patient was shifted and extubated in intensive care unit (ICU) after 4 hours of mechanical ventilation.

\section{Patient 2}

A 15-year-old girl reported to our hospital with a diagnosis of ostium secundum atrial septal defect
(ASD) with grade 2 dyspnea. On examination, the heart rate was $80 \mathrm{bpm}$, and the blood pressure was 120/70 mm $\mathrm{Hg}$. A TEE probe was placed after induction of general anesthesia and preoperative diagnosis confirmed. ASD closure was done by polytetrafluoroethylene membrane. An adequate de-airing of left atrium was done before tightening of the last suture for ASD patch closure. The CPB was terminated smoothly with the support of dobutamine $5 \mathrm{mcg} / \mathrm{kg} / \mathrm{min}$. The TEE examination revealed no residual ASD, normal biventricular function and an air pocket pooled in the anteroseptal region of the inter-ventricular septum (IVS) of LV (Fig. 4 and Video 3). A malignant VF was developed, 4 minutes after termination of CPB. Similar to the 1st case, air entering into the RCA was observed in ME LAX in TEE (Fig. 5 and Video 4 ) and the case was managed as RCA air embolism. Three direct cardioversion shocks of 10 Joules were delivered, in addition to administration of lignocaine $(1 \mathrm{mg} / \mathrm{kg})$. The systemic perfusion pressure was increased using vasopressor so as to increase coronary 


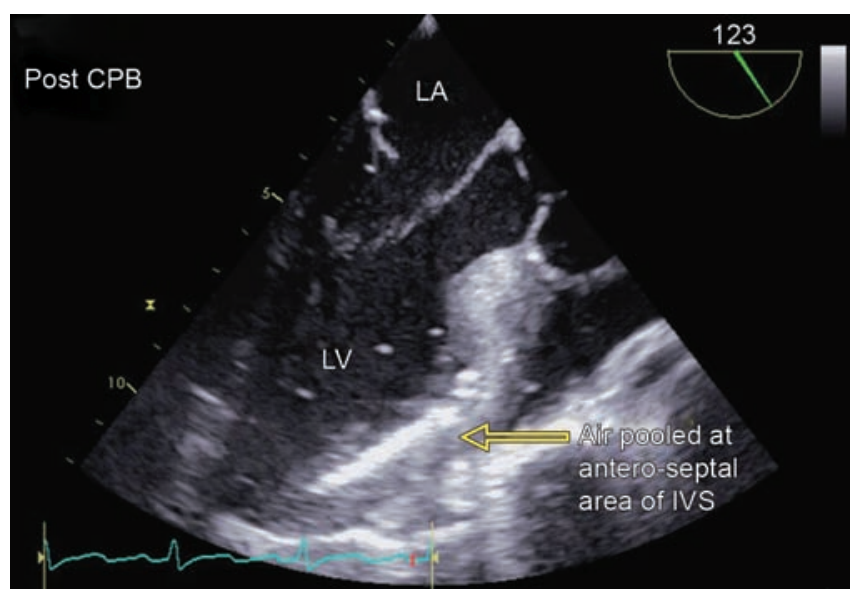

Fig. 4: Mid esophageal aortic valve long axis view showing air pooled on the anteroseptal region of interventricular septum (IVS) after towards left ventricle after cardiopulmonary bypass. (LA, left atrium; LV, left ventricle; RV)

perfusion, which helped in flushing the embolized air bubbles from coronaries. The RV function and ECG returned to baseline after successful management and the air pocket in the anteroseptal wall of LV gradually decreased. The patient was shifted and extubated after 2 hours in ICU without any further sequelae.

\section{DISCUSSION}

The air retained inside the cardiac chambers is a welldocumented phenomenon after cardiac surgeries, and it has been noted in 14 to $67 \%$ of patients undergoing $\mathrm{CPB}$ for valve replacement or CABG. $\cdot^{1-3}$ It can enter either into the right heart and/or the left heart chambers. Due to the risk of systemic air embolization, the air in left heart chambers is considered more dangerous than the right side. Effects of Intracoronary air embolism last for short periods and are often reversible. However, it can be lethal if not detected and managed in time. In our first case, air might have entered into the cardiac chamber due to (a) inadequate de-airing of the venous graft before unclamping or; (b) air entering the aortic root when it is punctured for the proximal anastomosis might have entered LV through mild aortic regurgitation. This hypothesis has been described in previous studies. ${ }^{2}$ Whereas, in the 2nd case, it was due to the opening of the right atrium.

Air most commonly settles on the nondependent areas of cardiac chambers as it floats on the blood. Anteroseptal area of IVS, interatrial septum, and left atrial appendage are some of the nondependent areas of left heart chambers. ${ }^{4}$

Due to the vast difference in acoustic properties between air and blood, air can be easily identified as a bright structure in TEE as it effectively reflects the ultrasound waves. ${ }^{5}$ TEE plays an important role in the identification of air and de-airing which is an important

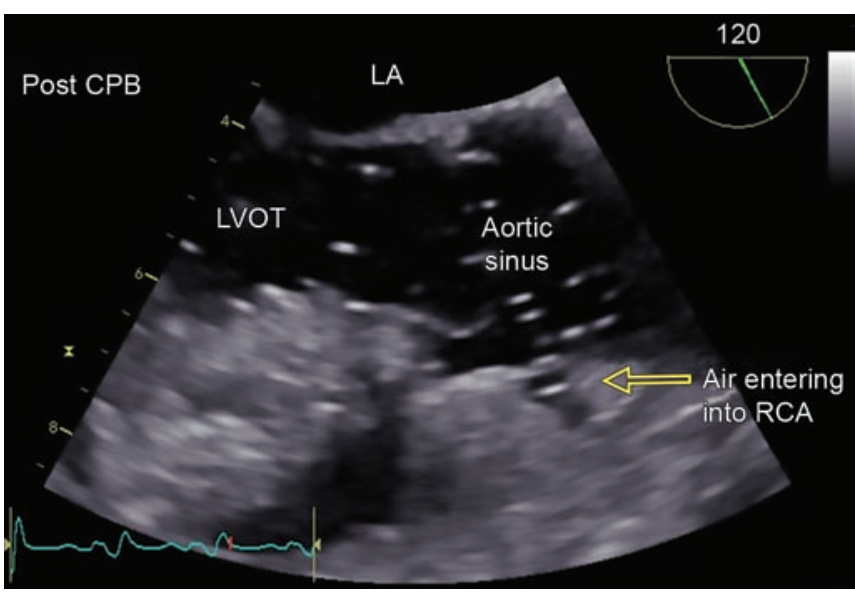

Fig. 5: Mid esophageal aortic valve long axis view showing A) the right coronary artery (RCA) before cardiopulmonary bypass (CPB), B) air entering into RCA after CPB (LA, left atrium; LVOT, left ventricular outflow tract)

step before coming out of $\mathrm{CPB}$ to avoid systemic embolization. During this process air can enter into the RCA, as the ostium of RCA is the most non-dependent part of aortic sinus in the supine position, leading to the development of RV ischemia and resultant STsegment changes, malignant arrhythmias, hemodynamic instability, right ventricular failure, and LV inferior wall hypokinesia. However, a similar type of signs and symptoms can occur in various conditions such as occlusion of the coronary artery because of a thrombus, spasm or entrapment of the RCA by sutures. ${ }^{6}$ Precise diagnosis of RCA air embolism is difficult in spite of being associated with a variety of hemodynamic and ECG changes. However, with the help of TEE, we can see the pooled pockets of air in various nondependent parts of $\mathrm{LV}$ and left atrium.

Chandraratna et al., ${ }^{3}$ diagnosed intracoronary embolism in two cases with the help of TEE by the sudden appearance of inferior wall akinesis with increased brightness of the area immediately after removal of the aortic cross-clamp. However, they have not observed the air entering into the RCA. Similarly, Lamm et al., diagnosed LAD artery air embolism based on TEE finding of increased brightness of the apical wall and severely hypokinetic left ventricle and apical akinesis with rapid spontaneous improvement. They also have not observed air entering into LAD. However, in both of our cases, we were able to observe the air entering into RCA. The echocardiographic visualization of air bubbles entering into RCA will confirm the diagnosis of intracoronary air embolism under circumstances of post-CPB sudden RV dysfunction, ST-segment elevation, and hemodynamic instability.

Intracoronary air embolism can be differentiated from mechanical obstruction of coronaries, myocardial scar, and myocarditis with the help of TEE. Both RWMA 
and increased relative myocardial brightness due to pooling of air especially in the anteroseptal region of $\mathrm{LV}$, suggestive of air, will be seen in cases with intracoronary air embolism. While in cases of coronary obstruction, myocardium will not appear bright. ${ }^{7}$ Increased localized myocardial brightness and RWMAs can be seen in patients with myocardial scar and myocarditis, but these abnormalities will be existing from the pre- $\mathrm{CPB}$ period. However, the RWMAs and bright myocardium appear in the post-CPB period in case of coronary air embolism. The RWMAs and bright myocardial appearance associated with intracoronary air embolism resolve spontaneously after further de-airing and increasing systemic perfusion pressures, but these measures will not be able to resolve such changes in patients with myocardial scarring and myocarditis.

In our cases, air embolism in RCA was initially suspected as one of the cause when the patient suddenly developed hemodynamic instability and malignant arrhythmias after coming off CPB. We confirmed the air embolism in RCA by observing air entering into RCA in ME LAX view. Presence of air bubbles and pools of air pockets in TEE were supportive pieces of evidence. Development of RV dysfunction and RWMA of the inferior wall of LV seen in TEE are surrogate manifestations of RCA air embolism.

The signs mentioned above and symptoms can occur in various conditions. Management will differ depending upon the etiology. Hence, accurate diagnosis of air embolism into RCA will help in specific management which includes delivering high Fio2, elevation of coronary perfusion pressure, infusion of the vasopressor, reinstitution of $\mathrm{CPB}$, and management of malignant arrhythmias. ${ }^{8}$ Some reported the use of adenosine to accelerate the clearance of the air bubble due to its vasodilatation property of coronaries. ${ }^{9}$

\section{CONCLUSION}

Right coronary artery air embolism is one of the common cause of hemodynamic instability after coming off CPB. The TEE helps in adequate and real-time de-airing of cardiac chambers, as well as in diagnosing intracoronary air embolism as the cause of sudden ST-segment elevation, hemodynamic instability, and RV dysfunction.

\section{REFERENCES}

1. Oka Y, Inoue T, Hong YO, Sisto DA, Strom JA, Frater RW. Retained intracardiac air. Transesophageal echocardiography for definition of incidence and monitoring removal by improved techniques. The Journal of thoracic and cardiovascular surgery. 1986 Mar;91(3):329-338.

2. Tingleff J, Joyce FS, Pettersson G. Intraoperative echocardiographic study of air embolism during cardiac operations. The Annals of thoracic surgery. 1995 Sep 1;60(3):673-677.

3. Chandraratna A, Ashmeg A, Pasha HC. Detection of intracoronary air embolism by echocardiography. Journal of the American Society of Echocardiography. 2002 Sep 1;15(9):1015-1017.

4. Bechtel A, Huffmyer J. Anesthetic Management for Cardiopulmonary Bypass. Seminars in Cardiothoracic and Vascular Anesthesia [Internet]. SAGE Publications; 2014 Apr 10;18(2):101-116.

5. Hammersley M. Deconstructing the qualitative-quantitative divide 1. Mixing Methods: qualitative and quantitative research [Internet]. Routledge; 2017 Jul 12;39-55.

6. Auer J, Ng CK. Surgical strategies for the treatment of chronic mitral valve insufficiency. Deutsche medizinische Wochenschrift (1946). 2000 Jul;125(28-29):888.

7. Lamm G, Auer J, Punzengruber C, Ng CK, Eber B. Intracoronary air embolism in open heart surgery-an uncommon source of myocardial ischaemia. International journal of cardiology. 2006 Oct 10;112(3):e85-86.

8. Geoghegan T, Lam CR. The Mechanism of Death from Intracardic Air and Its Reversibility. Annals of surgery. 1953 Sep;138(3):351-359.

9. Yew KL, Razali F. Massive coronary air embolism successfully treated with intracoronary catheter aspiration and intracoronary adenosine. International journal of cardiology. 2015 Jun 1;188:56-57. 